\title{
Research on the Optimization Coupling for Enhancing Polymer Optical Fiber Communication
}

\author{
Zhengqin Dong ${ }^{1, *}$ and Yun $\mathrm{Lu}^{2}$ \\ ${ }^{1}$ School of Computer Science and Technology, Wuhan University of Technology, Wuhan, China, 430074 \\ ${ }^{2}$ School of Naval Architecture and Ocean Engineering, Huazhong University of Science and Technology, Wuhan, China, 430074 \\ * Corresponding author: 15926424120@163.com
}

\begin{abstract}
Aiming at improving the performance for optical fiber communication, the various factors to enhance the optical fiber bandwidths are discussed in detail. A novel design with cost effective coupling is explored. The proposed package with the integrated signal conditioners will make the transceiver packages simple and compact. This signal conditioner also helps in effective optical coupling without the need of any additional light. This connector less package design, universal to any light source proposes to enhance the data rate and is widely manufacturing at an ease of installation and low cost.
\end{abstract}

\section{Introduction}

In the rising data rate market, short distance communication requires higher bandwidths and low cost components. One of the potential areas that demands high data rate is premise networks like broadband access network and home automation. POFs are being considered as a practical solution for this kind of application. Their major advantages include a very flexible structure, lower connection costs and immunity to electromagnetic interference. The large core structure of POF is advantageous in installation while it allows a large number of modes to propagate through the core.

The presence of multimode limits the bandwidth of POF due to intermodal dispersion. The bandwidth enhancement studies have got foremost interest to keep POFs attractive in the growing data rate market. The three major component's characteristics, say transmitter, fiber and receiver, decide the bandwidth capacity of a multimode fiber link. Since these networks are implemented in premises and local area networks, overall cost of the components is also of major concern. Even though LED sources are cost effective transmitters, the requirement for higher bandwidths made them less attractive for POF communications [1-2]. The high power, high data rate, narrow spectral width and smaller spot size compared to LEDs raised low cost $850 \mathrm{~nm}$ VCSEL as an alternative solution for such applications. Connector less packages and fiber plug in modules also helped to maintain POF system installation cost lower. There are new generations of POF, which offer a higher bandwidth are manufactured by engineering the core index profile. A graded index (GI) POF can transmit four times higher data rates than a step index POF. But they are offered at higher price than normal POFs.

Another alternative to increase the bandwidth of POF is by external coupling mechanisms, which selectively excites either the fundamental modes lying at the center of the core or higher order modes lying towards the cladding. One of the widely used methods to achieve this condition is attaching a mode conditioning single mode fibers with the multimode fiber. But this will have the additional process of splicing or connecting the SMF to POF [3].

\section{The Model Bandwidth}

The bandwidth of a fiber is decided by the aggregate performance of all the modes in the fiber or in other words the modal bandwidth depends on the number of modes excited in the MMF. The optical launch conditions have a greater effect on deciding the number of modes excited. The lower order modes are centered on the core, while a higher order mode finds their paths towards the cladding. Over filled and under filled launches are the two general terms associated with the light propagation through fiber. In the former case the light launched in to the fiber over fills the core of the fiber and hence excites the maximum number of modes possible. In the latter case, the light launched fills only a certain portion of the fiber and excites limited number of modes. Restricting the modal excitation by means of an undefiled launch condition is known as restricted mode launch (RML) [4]. Figure 2 shows the example of overfilled and under filled launches. 


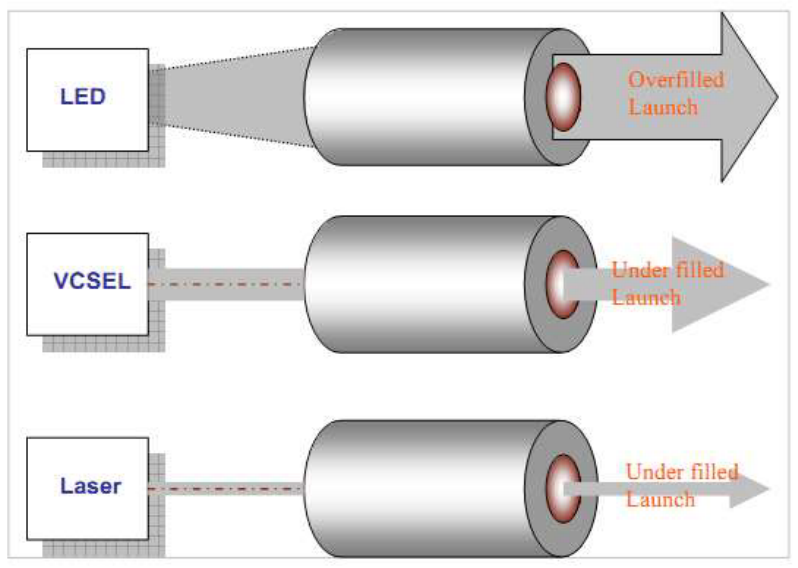

Fig. 1. The example of overfilled and under filled launches.

The RML can be either center launch or offset launch. In the center launch, the optical power from a single mode fiber or laser is coupled to the center of the multimode fiber. This excites the lower order modes, fundamental mode, centered at the core. Alternatively in the offset launch, the power is launched to an area offset from the center of the fiber. In this method the power is coupled to the higher order modes having relatively low modal dispersion.

Both these methods have proven very effective in increasing the bandwidth of multimode fibers by controlling the number of modes excited. But the fibers having discontinuities in their refractive index profile near the center badly affects the performance under center launch scheme. The variation in refractive indices offers different speeds to light and ends up in the phenomena known as Differential Mode Delay (DMD), which considerably reduces the effective bandwidth. In an offset launch, the launch position can be selected to avoid this defective region.

The equilibrium mode distribution (EMD) and steady state mode distribution are the other two concepts related to light launch conditions. EMD is the mode distribution obtained after going through a system, where the output mode distribution is unaffected by the input mode distribution; independent of launch conditions. This situation is seldom met [5].

Steady state distribution is reached when the mode distribution is no longer changes along the fiber. This depends on launching conditions. The maximum steady state is reached in an over filled launch condition. In the absence of strong mode coupling, restricted launch condition also creates a steady state mode distribution. Once the steady state is reached, the transfer of energy between the modes will stop.

Mode stripping is another method used to reduce the number of higher order modes propagating in a fiber and thereby improving the bandwidth further. They are of two types: core mode filter and cladding mode stripper. In the core mode filter the fiber is wound over a mandrel to reduce the higher order modes in the core. During this process the higher order modes leaks to the cladding and is being lost. This results in an exit numerical aperture less than the material NA of the fiber. A fiber that has reached modal equilibrium, along with the reduced NA, is said to exhibit longlaunch conditions. Cladding mode stripper is required in short distance applications since the higher order modes leaked to the cladding couples back to the detector at the receiver side. It is necessary to remove this mode using a cladding mode stripper. The cladding mode stripper is nothing but a higher refractive index material coated on the fiber cladding. Here the cladding buffer is removed initially and coated with a higher index material. Fig. 2 shows the equilibrium steady state mode propagation.

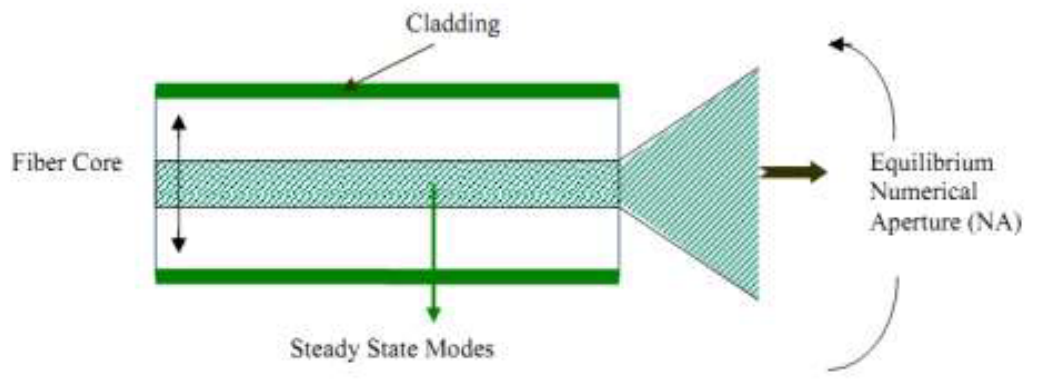

Fig. 2. Equilibrium steady state mode propagation. 


\section{Experiment Results and Discussion}

To verify the effects of light source parameters on modal distribution, we set up an experiment to launch the light into POF having a core diameter $980 \mu \mathrm{m}$. In the initial experiment, light from a $1310 \mathrm{~nm}$ laser diode is launched to the POF using a single mode fiber of core diameter $9 \mu \mathrm{m}$ and the near field patterns are captured by a beam analyzer. The second set up replaced the single mode fiber with a multimode fiber of $62.5 \mu \mathrm{m}$ to repeat the experiment.

The design of RML coupling requires the information on encircled flux (EF) of the light source used. The encircled flux is the percentage of the power within a given radius when light is launched by a transmitter into a multimode fiber. The modal distribution of the fiber depends on the numerical aperture and spot size at the launch end of the fiber. If the spot size is small and its NA is less than the fiber core, the optical power will be concentrated at the center of the fiber core. Figure 3 shows the experimental setup for optimizing the launch conditions and figure 4 (a) shows the near field output pattern of POF, when light launched with MMF and (b) shows the launched with an SMF.

The bigger spot size and larger NA, leads to higher order mode excitation. Hence in any optics design for mode control it should take care of a matching spot size and NA of the source and fiber. A steady state equilibrium mode distribution state can be realized by an identical far and near field patterns. The radiation patterns are compressed in the case of RML conditions and enlarged in the case of OFL conditions.

Keeping in mind the necessity of a cost effective compact system, we explored the possibility of integrating the signal conditioning elements inside the package. In the proposed design, at the transmitter side the molded plastic package consists of a restricted mode launcher and at the receiver side it involves a mode stripper. Restricted mode launcher, which helps to keeps the encircled flux of the source to remain the same irrespective of the light sources used. The design of this element is optimized to generate the same effect of launching as with a single mode fiber of NA 0.1 . The signal conditioner at the transmitter side provides center launch with undefiled launching conditions. This helps to excite only the lowest order modes and hence reduces the modal dispersion. The concept of mode stripper is implemented in such a way that it not only helps to radiate the higher order modes excited but also couples the light effectively to the detector. The dimensions of the signal conditioners are optimized based on the optical simulations with a VCSEL source of divergence half angle 160, SI POF having a core diameter of $980 \mu \mathrm{m}$ and a detector radius of $50 \mu \mathrm{m}$.

Based on the theoretical calculations, POF of $980 \mu \mathrm{m}$ dimensions will support thousands of modes in over filled condition. The design of RML is done to reduce the number of modes excited to a minimum by concentrating the light only to excite the fundamental mode. There by reducing the modal dispersion and increasing the modal bandwidth. The mode stripper at the receiver side is designed to eliminate higher order modes and have efficient coupling to the detector. The mode stripper design is such a way that it will eliminate minimum $10 \%$ higher order modes. So with the help of both RML and Mode stripper, the data rate will be enhanced considerably. The fabrication of this integrated components are proposed to do with plastic molded packages. The transmitter and receiver plastic packages can be fabricated with optimized signal conditioner structure cavities. These cavities are then filled with optically transparent epoxy of suitable index of refraction. Either ultra violet curing or thermal curing can be used for reinforcing the structure. It is better to use an epoxy having $\mathrm{Tg}>95 \mathrm{C}$ having a shrinkage rate less than $2 \%$ of the initial volume.

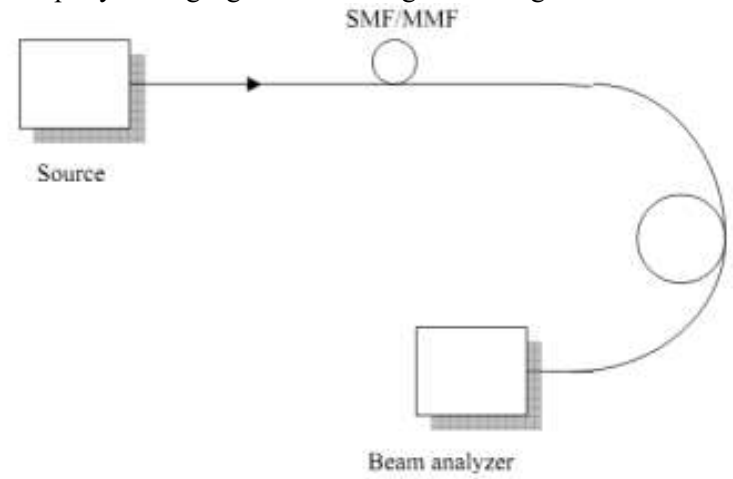

Fig. 3. Experimental setup for optimizing the launch conditions. 

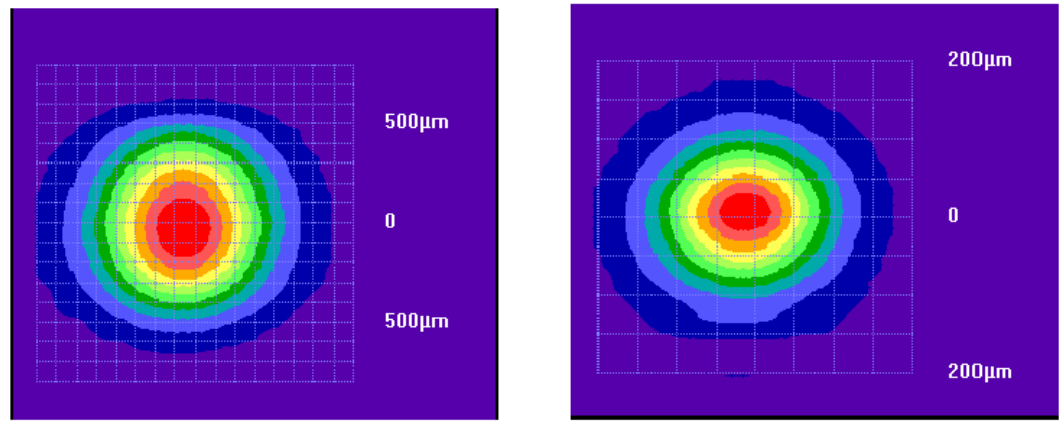

Fig. 4. (a) Near field output pattern of POF when light launched with MMF(b) launched with an SMF.

\section{References}

1. Wu He. Examining students' online interaction in a live video streaming environment using data mining and text mining. Computers in Human Behavior, (2013), pp. 291-304.

2. Fu Xiao, Cheng Fan. Data mining in building automation system for improving building operational performance. Energy \& Buildings, (2014), pp. 75-83.

3. Benrun Huang, Li Xia, Zhengguo Wu, Weiping Zhou. Control Strategy of A Dynamic Voltage Restorer (DVR) Based on Line Voltage Compensation. Energy Procedia, (2011), pp. 13-26.

4. Wei Dai, Mostafa Bassiouni. An improved task assignment scheme for Hadoop running in the clouds. Journal of Cloud Computing, (2013), pp. 21-33.

5. Wei Kuang Lai, Yi-Uan Chen, Tin-Yu Wu, Mohammad S. Obaidat. Towards a framework for large-scale multimedia data storage and processing on Hadoop platform. The Journal of Supercomputing, (2014), pp. $681-696$. 